\title{
Desajuste de trayectorias estudiantiles. Caso comparativo de la Universidad Federal de Paraíba y la Universidad de València
}

\author{
Adriana Santos Diniz, Alícia Villar, Francesc \\ Hernàndez \& Uyguaciara Castelo Branco
}

\begin{abstract}
Resumen
Este artículo aborda la cuestión del ajuste académico en las trayectorias universitarias entendiéndolo en base al ritmo de renovación de los estudiantes y de idoneidad de su rendimiento académico. El objetivo planteado es calibrar el ajuste entre ambos indicadores de rendimiento, es decir, renovación e idoneidad para poder proponer una medida integral de ajuste. Proponemos una metodología para ponderar el grado de ajuste en las trayectorias universitarias a partir de los indicadores de renovación e idoneidad mediante la propuesta de un nuevo indicador que denominamos tasa de ajuste. El procedimiento de su cálculo es a través de la adaptación del habitual índice de Gini y cuenta con la característica que permite aplicarlo a diferentes unidades de análisis (titulación, áreas de conocimiento, universidad). En la aplicación que presentamos en este artículo se describe la comparación realizada entre la Universidad Federal de Paraíba (Brasil) y la Universidad de València (España). Los resultados de este estudio permiten dar respuesta al objetivo planteado y proporcionar una dimensión del ajuste de las trayectorias académicas en las titulaciones de ambas instituciones universitarias. Esta propuesta permite observar a las instituciones universitarias según su grado de ajuste de acuerdo con los ritmos de rendimiento académico esperados a nivel institucional.
\end{abstract}

Palabras clave:

universidad; indicadores; rendimiento; renovación; idoneidad. 


\title{
Desajuste em trajetórias de estudantes. Caso comparativo entre a Universidade Federal de Paraíba e a Universidade de València
}

\begin{abstract}
Resumo: Este artigo aborda a questão do ajuste acadêmico nas trajetórias das universidades, entendendo-o com base no ritmo de renovação estudantil e adequação de seu desempenho acadêmico. O objetivo é calibrar o ajuste entre os dois indicadores de desempenho, ou seja, renovação e adequação para poder propor uma medida de ajuste integral. Propomos uma metodologia para ponderar o grau de ajuste nas trajetórias das universidades com base nos indicadores de renovação e adequação por meio da proposta de um novo indicador que chamamos de taxa de ajuste. O procedimento de seu cálculo é através da adaptação do índice de Gini usual e tem a característica que permite que seja aplicado a diferentes unidades de análise (graduação, áreas de conhecimento, universidade). A aplicação apresentada neste artigo descreve a comparação feita entre a Universidade Federal de Paraíba (Brasil) e a Universidade de València (Espanha). Os resultados deste estudo nos permitem responder ao objetivo declarado e fornecer uma dimensão do ajuste das trajetórias acadêmicas em cursos de graduação de ambas instituições universitárias. Essa proposta permite observar as instituições universitárias de acordo com seu grau de ajustamento de acordo com as taxas de desempenho acadêmico esperadas no nível institucional.
\end{abstract}

Palavras-chave: universidade; indicadores; desempenho; renovação; adequação.

\section{Désajustement dans les trajectoires des étudiants. L'affaire comparative entre l'Université Fédérale de Paraíba et l'Université de Valence}

Résumé: Cet article aborde la question de l'adaptation académique des trajectoires universitaires sur la base du taux de renouvellement des étudiants et de l'adéquation de leurs performances académiques. L'objectif est d'étalonner l'ajustement entre les deux indicateurs de performance, c'est-à-dire le renouvellement et l'adéquation, afin de pouvoir proposer une mesure intégrale de l'ajustement. Nous proposons une méthodologie permettant d'évaluer le degré d'ajustement des trajectoires universitaires sur la base des indicateurs de renouvellement et d'adéquation. Pour ce faire, nous proposons un nouvel indicateur appelé taux d'ajustement. La procédure de calcul se fait par l'adaptation de l'indice de Gini habituel et présente la caractéristique qui permet de l'appliquer à différentes unités d'analyse (degré, domaines de connaissance, université). Dans l'application que nous présentons dans cet article, nous décrivons la comparaison faite entre l'Université Fédérale de Paraíba (Brésil) et l'Université de València (Espagne). Les résultats de cette étude nous permettent de répondre à l'objectif énoncé et de donner une dimension à l'ajustement des trajectoires universitaires à les degrés des établissements universitaires. Cette proposition permet d'observer les institutions universitaires en fonction de leur degré d'ajustement d'accord avec des rythmes de performance académiques attendus au niveau institutionnel.

Mots clés: université; indicateurs; performance; rénovation; adéquation.

\section{Misadjustments Index in students' trajectories. The comparative case between the Federal University of Paraíba and University of Valencia}

\begin{abstract}
This article approaches the issue of academic adjustment in the university pathways, understanding it based on the rate of student renewal and the suitability of their academic performance. The objective is to measure the adjustment between both performance indicators, that is, renewal and suitability to be able to propose an integral measure of adjustment. We propose to evaluate the degree of adjustment in university based on the indicators of renewal and adequacy through the proposal of a new indicator that we call the adjustment rate. The calculation procedure is through the adaptation of the usual Gini index and has the characteristic that it can be apply to different units of analysis (degree, areas of knowledge, university). In the application that we present in this article we describe the comparison made between Federal University of Paraíba (Brazil) and University of Valencia (Spain). The results of this study allow us to respond to the stated objective and provide a dimension of the adjustment of the academic trajectories in the degrees of the both university institutions. This proposal allows to observe university institutions according to their degree of adjustment in accordance with the academic performance expected at the institutional level.
\end{abstract}

Keywords: university; indicators; achievement; renovation, suitability. 


\section{Introducción}

Este trabajo se ubica en el estudio de las trayectorias académicas en educación superior, una temática de investigación consolidada e interdisciplinaria que cuenta en los últimos años con numerosos trabajos de alcance internacional. Son diversas las maneras de desarrollarse las trayectorias y de vincularse a los estudios universitarios, así como de integrarse en la universidad, una institución con sus procesos y particularidades que ha ido evolucionando en las últimas décadas, tal y como ha sido desarrollado en obras de referencia desde la sociología de la educación. En el conocido trabajo de Los herederos de Pierre Bourdieu y Jean-Claude Passeron (1964), ya se apuntaban las diferencias existentes para acceder a la universidad según el origen social de los estudiantes, señalando las desiguales probabilidades entre los estudiantes de clase obrera, de clase media y de clase alta. La educación universitaria es, por tanto, selectiva y selecciona a unos elegidos, a unos "herederos". En los últimos años se han realizado investigaciones relevantes en el ámbito de la sociología de los estudiantes universitarios, como las desarrolladas por Alain Coulon $(1997,2005)$ que aporta la noción de "afiliación" (affiliation) en la configuración de lo que denomina "el oficio del estudiante" o por George Felouzis $(2001,2003)$ quien destaca el aumento de la diversificación en el perfil del estudiantado y califica a la universidad como una "institución débil" en cuanto a la aplicación de normativa. Retomando la idea de la herencia cultural de Bourdieu y de los denominados "herederos", Valérie Erlich estudia los "nuevos" estudiantes en la universidad francesa señalando la bipolarización entre el modelo de los "herederos" y el de "nuevos" estudiantes con "un futuro profesional que no está asegurado" (Erlich, 2004: 487).

En el contexto inglés destacamos las investigaciones desarrolladas por la socióloga Rachel Brooks sobre las transformaciones de los estudiantes universitarios. En una reciente investigación se ha comparado las políticas de seis países europeos, destacando que continúa existiendo una heterogeneidad importante entre los países a la hora de comprender al estudiante universitario (Brooks, 2019). Esta autora también ha señalado la configuración de discursos dominantes que afectan a la configuración del estudiante universitario a través de expresiones como "futuro trabajador" (future worker) y "trabajador duro" (hard- worker) que se relacionan con el reposicionamiento de la educación superior entendida como un bien económico (Brooks, 2018: 746).

En las últimas décadas el sistema de educación superior sufrió profundos cambios, de carácter cuantitativo y cualitativo, con una marcada democratización y masificación del sistema, en diferentes casos, como en España y en Brasil. En España se ha venido destacando en los trabajos especializados sobre universidad y estudiantes (Segrera, 2008). En el estudio dirigido por Antonio Ariño (2008) se presenta una tipología de estudiantes en relación a la vinculación a los estudios destacando un "compromiso 
flexible" entre los estudiantes. En este y en otros trabajos continúan destacando la importancia del origen social (Daza, Troiano y Elias, 2019).

En el caso de Brasil, se ha venido desarrollando estudios sobre la expansión/ democratización de la educación superior y sus reflejos para los procesos de igualdad/desigualdad, inclusión/exclusión, permanencia/abandono (Dias Sobrinho y Días, 2008; Jezine y Bittar, 2013; Sguissardi, 2015; Chaves, Maués y Hage, 2016). El caso específico de Paraíba, ubicado en la región nordeste del país, el Núcleo de Estudios e Investigaciones en Educación y Sociedad de la Universidade Federal de Paraíba, dirigido por Edineide Jezine, ha propuesto una Escala para Evaluación de la Permanencia Discente (Nakamura, Castelo Branco y Jezine, 2016; Castelo Branco, Nakamura y Jezine, 2017). Los resultados indican que la permanencia es un proceso relacionado a las convicciones del estudiante, desde antes del ingreso en la universidad, como las motivaciones para elegir el curso y las expectativas del estudiante respecto al mercado laboral. Los estudiantes no perciben las condiciones estructurales de la universidad y los programas de asistencia de becas como importantes para su permanencia.

En este artículo se pretende contribuir a esa línea de investigación sobre universidad y trayectorias de estudiantes universitarios partiendo del objetivo de calibrar el ajuste entre unos indicadores relacionados con la graduación para poder proponer una medida integral que hemos denominado Índice de Desajuste Académico (IDA). A partir de ese objetivo, proponemos una metodología para ponderar el grado de ajuste (o de desajuste) en las trayectorias universitarias a partir de indicadores de renovación e idoneidad. Entendemos por ajuste o desajuste académico la fluidez del conjunto de trayectorias del estudiantado en un espacio y un tiempo determinado, con referencia al período que se considera normal de duración de los estudios, así como de otros comportamientos académicos esperados institucionalmente. Así definido, el ajuste integra otras variables (como la renovación o la idoneidad, entre otras), permite comparaciones de base heterogénea (como la comparación entre distintas universidades o dentro de una misma universidad, entre distintas titulaciones). Considerar el ajuste/desajuste académico en el análisis de las trayectorias de los estudiantes, así como también en los procesos de vinculación hacia los estudios (como la asistencia a clases o a los exámenes, entre otros) ofrece, también, poder detectar y analizar los obstáculos y resistencias sistémicas que encuentran los sujetos en sus trayectorias.

En el siguiente apartado concretaremos un poco más en la temática de las trayectorias educativas universitarias en cuanto a los fenómenos de renovación e idoneidad académica. Serán analizados de modo comparado datos de la Universidad de València (UV) y de la Universidade Federal de Paraíba (UFPB). 


\section{Trayectorias en Educación Superior: renovación e idoneidad académica}

En las dos últimas décadas han proliferado los intentos de comparar internacionalmente indicadores de rendimiento educativo. En un momento reciente de crisis económica, especialmente en los países del Sur de Europa, como España, Portugal, Italia o Grecia, existe una presión mayor para aumentar la eficiencia de las inversiones públicas y evitar cualquier desperdicio de recursos (Villar, Vieira, Hernàndez y Nunes, 2012), generalizándose las cuantificaciones del rendimiento educativo mediante indicadores, como la tasa de eficiencia o la tasa de abandono, entre otros, y que se han aplicado para los procesos de verificación de la calidad de los grados universitarios dentro de los nuevos procesos del Espacio Europeo de Educación Superior.

En el caso del abandono universitario, tal y como hemos estudiado desde hace unos años y sobre el que hemos publicado varios trabajos, partíamos de un cuestionamiento fundamental y necesario: el abandono universitario no puede considerarse siempre como total, es decir, no puede entenderse, ni medirse, como un abandono del sistema universitario. De hecho, según nuestros cálculos, aquello que el indicador medía era más bien un cambio de titulación. Este hecho lo comprobamos tanto en el caso español, como portugués (Villar, Vieira, Hernàndez y Nunes, 2012). En trabajos posteriores dedicados a este cambio de itinerario, conceptualizado como reubicación académica, hemos demostrado que estos cambios, lógicamente, están matizados por el momento en que se producen (especialmente, tras el primer curso), que son más intensos en unas áreas de conocimiento (por ejemplo, más intensos en las ingenierías) o por otros factores relevantes, como el sexo, la edad del estudiante o si se trata de estudiantes que combinan trabajo con estudios (es un fenómeno más masculinizado, más probable en estudiantes de mayor edad y en aquellos que estudian y trabajan). Estos resultados nos llevan a matizar las reubicaciones académicas con el fin de entenderlas mejor (Villar, Hernàndez y García-Ros, 2017).

Por tanto, esta referencia a nuestra línea de investigación sobre reubicaciones universitarias que se desarrolla a partir de un cuestionamiento del indicador y concepto de abandono nos lleva a plantear un cuestionamiento similar en el caso que tratamos en este trabajo sobre la idoneidad en la trayectoria estudiantil. En este sentido, en la universidad actual los itinerarios formativos no transcurren necesariamente de manera lineal, ni unívoca, ya que se desarrollan itinerarios formativos de retorno, de reubicación, como hemos expuesto en anteriores trabajos (Villar, Hernàndez y García-Ros, 2017). La diversidad en el perfil del estudiante universitario ha comportado maneras distintas en su vinculación a los estudios y al hecho de llegar y estar en la universidad. Este conocimiento de la realidad nos permite partir de un cuestionamiento inicial sobre lo que significa "idoneidad" del rendimiento académico. La tasa de idoneidad es un indicador acordado e incluido en el sistema de indicadores universitarios del sistema español 
para cuantificar cómo de "idónea" es la trayectoria académica de los estudiantes. Esa idoneidad hace referencia al tiempo teórico de duración de una carrera universitaria, es decir que, si el plan de estudios de un grado está estructurado en cuatro años de duración, lo idóneo, según se entiende y mide mediante esta tasa, es graduarse en cuatro años o incluso antes.

Por otra parte, la tasa de renovación es uno de los indicadores relacionados con el proceso académico de los estudiantes universitarios y sus resultados. La tasa de renovación forma parte del conjunto de indicadores de graduación entre los cuales también se encuentra la tasa de idoneidad, de graduación o, entre otros, de eficiencia. La renovación hace referencia a la proporción de estudiantes de una titulación que son de nuevo ingreso en el curso académico considerado.

Así pues, en los apartados siguientes utilizamos datos de la tasa de idoneidad, y de la tasa de renovación. Cabe avanzar aquí que la manera de entender esa "idoneidad" de la trayectoria estudiantil nos plantea el mismo cuestionamiento al que ya nos enfrentamos en trabajos anteriores sobre el abandono universitario. Consideramos que esa "idoneidad" debería ser revisada para poder incorporar una realidad universidad más diversa y situaciones diferentes a las que tienen que hacer frente los estudiantes y que, lógicamente, condicionan su ritmo de estudio.

\section{Fuentes de datos y metodología}

Partimos del objetivo de determinar un índice para medir el desajuste en las trayectorias universitarias, esto es, una herramienta indicadora que permita comparar el tránsito del estudiantado de educación superior entre dos instituciones (universidades, facultades), entre dos tipos de estudios (grados o postgrados) o, incluso, entre desagregaciones como son las trayectorias diferenciadas de hombres y mujeres, por ejemplo. De este modo, los desajustes se plantean matemáticamente como un problema de flujo, porque se trata de averiguar cómo «fluye» el estudiantado en unos casos y otros. Para ello, definiremos, en primer lugar, una "tasa de fluidez» y a continuación construiremos el que denominaremos Índice de Desajuste Académico (IDA).

Las fuentes utilizadas son los datos proporcionados por el Servicio de Informática para el caso de la UFPB y las estadísticas del Sistema Integrado de Información Universitaria del Ministerio de Educación en el caso de la UV.

Para formular la tasa de fluidez partiremos de dos tasas que ya se utilizan en el sistema de indicadores universitarios: la tasa de idoneidad y la tasa de renovación, que comentaremos a continuación. La tasa de idoneidad (TI) es uno de los indicadores utilizados en el Sistema de Indicadores Universitarios en el caso español. Se define como el porcentaje de estudiantes de nuevo ingreso en el estudio en un curso que finalizan el estudio inicial en el curso de finalización teórico o antes. 
Supongamos un grado o titulación cualquiera, cuya duración teórica es de K cursos. En el año A se matriculan unos estudiantes nuevos (que denominaremos $\mathrm{N}$ ) respecto del total de los que se matriculan (M). Es trivial que $M \geq N$. La tasa de renovación (TR) es, según la definición habitual y con nuestras siglas, la proporción de N sobre M. Así que se formula así:

$$
T R=\frac{N}{M} \quad[1]
$$

Hay que advertir que $\mathrm{N}$ no tiene por qué coincidir con los estudiantes de primer curso, porque puede haber estudiantes que se incorporen en cursos posteriores (por ejemplo, porque procedan de otro centro universitario) o que se vuelvan a matricular en primer curso (o en la mayoría de sus créditos), porque no han conseguido aprobar las materias. Pues bien, si consideramos el conjunto de estudiantes de primer curso (que denominaremos $\mathrm{P}$ ), también podemos preguntarnos cuántos de ellos habrán concluido en el año $A+K$, y el número de estos se denominará $Q$. Está claro que $P \geq Q$. Pues bien, se denomina tasa de idoneidad $(\mathrm{TI})$ a la proporción de $\mathrm{Q}$ respecto de $\mathrm{P}$.

$$
T I=\frac{Q}{P} \quad[2]
$$

Consideramos, entonces, que la "fluidez" en el tránsito del estudiantado por un grado o titulación tiene que ver con ambas tasas, con la idoneidad y con la renovación. Es por ello que definiremos una tercera tasa, la tasa de fluidez (TF), como el producto de ambas. Así tenemos que (según las fórmulas [1] y [2]):

$$
\mathrm{TF}=\mathrm{TR} * \mathrm{TI}=\frac{N}{M} * \frac{Q}{P}=\frac{N Q}{M P}
$$

Los datos de la Universidad de València se recogen en la tabla 1. Los de la Universidad Federal de Paraíba en la tabla 2. 
Tabla 1 - Renovación, idoneidad y fluidez en la Universidad de València en hombres y mujeres.

\begin{tabular}{|c|c|c|c|c|c|c|c|c|c|}
\hline \multirow{2}{*}{ TITULACION } & \multicolumn{3}{|c|}{ TASA.DE-RENOVACION.(TR)a } & \multicolumn{3}{|c|}{ TASA-DE·IDONEIDAD-(TI) $a$} & \multicolumn{3}{|c|}{ TASA.DE-FLUIDEZ(TF) $\alpha$} \\
\hline & Ambosa & Hombresc & Mujeress & Ambosa & Hombresa & Mujeresa & Amboss & Hombress & Mujereso \\
\hline Totalo & 4,40 & $24,7 \propto$ & $24,3 \alpha$ & 46,20 & 37,30 & 52,10 & 11,30 & 9,20 & 12,70 \\
\hline Total-Educacióno & $5,4, \alpha$ & $25,8 \mathrm{a}$ & 25,30 & 68,00 & 59,700 & 70,10 & $17,3 x$ & $15,4 \alpha$ & $17,7 x$ \\
\hline $\begin{array}{l}\text { Formación -de-docentes de } \\
\text { enseñanza-infantila }\end{array}$ & $26,7 x$ & 29,10 & 26,50 & 68,20 & $* * * \alpha$ & $68,5 \propto$ & 18,20 & $* * * \alpha$ & 18,2 \\
\hline $\begin{array}{l}\text { Formación de docentes de- } \\
\text { enseñanza-primariad }\end{array}$ & $25,8 \mathrm{a}$ & 27,20 & 25,20 & 71,50 & 63,700 & 75,20 & $18,5 \times$ & $17,3,0$ & 19,0 \\
\hline $\begin{array}{l}\text { Otra-Formación-de personal- } \\
\text { docente-y-ciencias-de-la- } \\
\text { educacióna }\end{array}$ & 23,30 & $20,7 a$ & 23,90 & $61,0,0$ & $41,5 \propto$ & $64,4 x$ & 14,20 & 8,60 & 15,4 \\
\hline Total Artes $\mathrm{y}$ humanidades $\alpha$ & 23,50 & 24,10 & 23,10 & $37,5 \infty$ & 32,60 & $40,7 x$ & 8,80 & 7,900 & $9,4 \mathrm{a}$ \\
\hline $\begin{array}{l}\text { Técnicas audic } \\
\text { medios de-com }\end{array}$ & 23,900 & 23,00 & 24,50 & 65,30 & 63,300 & $66,7 x$ & 15,60 & 14,60 & 16,30 \\
\hline Artes:a & $23,8 \mathrm{a}$ & $27,3 x$ & 220 & 33,30 & 32,80 & $33,7 \mathrm{a}$ & 7,90 & 8,90 & 7,40 \\
\hline Lenguaso & 24,40 & $25,8 \mathrm{a}$ & $24 \alpha$ & 41,00 & 37,60 & 42,20 & $10,0,0$ & 9,70 & 10,10 \\
\hline Humanid & $21,8 \mathrm{a}$ & $22,4 \mathrm{a}$ & 20,80 & 26,40 & 24,50 & 30,10 & 80 &, $5 \mathrm{a}$ & 6,30 \\
\hline $\begin{array}{l}\text { Total-Ciencia } \\
\text { derechoo }\end{array}$ & 23,10 & 23,50 & $22,8 a$ & 42,50 & 36,500 & $46,3 \mathrm{a}$ & 9,80 & 8,60 & 10,60 \\
\hline Psic & $22,4 \mathrm{a}$ & 23 & 2 & 48,30 & $43,9 a$ & $50,0 x$ & 10,80 & 10,10 & 11,10 \\
\hline Economíad & 19,10 & $20,8 x$ & $17,0 \mathrm{xa}$ & $34,8 x$ & 29,50 & $43,1 \alpha$ & 6,60 & 6,10 & 7,30 \\
\hline $\begin{array}{l}\text { Otras Cienci } \\
\text { comportamie }\end{array}$ & $24,3 \mathrm{a}$ & 22,80 & $25,7 \mathrm{a}$ & 39,30 & $35,7 \alpha a$ & 41,90 & 9,50 & 8,10 & 10,80 \\
\hline formacióna & $24 \alpha$ & $20,1 \times 1$ & 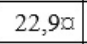 & 41,20 & $a$ & $43,5 \mathrm{a}$ & 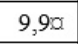 & $\alpha$ & 0 \\
\hline ación $y \cdot g e s t i o ́ n \cdot d e-$ & $24,7 x$ & 24,20 & $25,1 \mathrm{a}$ & 44,50 & 37,30 & $51,7 x$ & $11,0 \times$ & $9,0: 0$ & 13,00 \\
\hline $\begin{array}{l}\text { ción } \text { comercial-y. } \\
10\end{array}$ & 32,10 & 31,90 & $32,2 \alpha$ & $31,3 \alpha$ & $5,8 \mathrm{a}$ & $36,1 \propto$ & 10,10 & 8,20 & 11,60 \\
\hline Derechoo & $20,4 \propto$ & 220 & 19,30 & 33,10 & $27,9 x$ & $36,8 \mathrm{a}$ & $6,7 \alpha$ & 6,10 & 7,10 \\
\hline Total-Ciencias e e infor & $22,0: 0$ & 23,30 & $20,4 \propto$ & $31,7, \alpha$ & 32,40 & $30,9: 0$ & 7,000 & 7,60 & 6,30 \\
\hline Ciencias-de-la-vidad & 21,40 & 230 & 20,10 & 45,60 & 47,20 & 44,30 & 9,800 & 10,90 & 8,90 \\
\hline $\begin{array}{l}\text { Ciencias Físicas, químicas, } \\
\text { geológicasa }\end{array}$ & $21,3 \alpha$ & $23 a$ & 19,20 & 18,60 & 22,30 & $12,5 \propto$ & $4,0 \propto$ & 5,10 & 2,40 \\
\hline Matemáticas $\mathrm{y} \cdot$-Estadísticaa & 24,20 & 260 & 21,90 & 27,60 & 30,00 & $23,7 x$ & 6,700 & $7,8 \propto$ & 5,20 \\
\hline Informáticad & 23,60 & 22,60 & $28,7,0$ & 17,10 & 15,90 & $* * * 0$ & $4,0: 0$ & 3,60 & $* * *$ \\
\hline Ingenieríasa & $27,7 \times$ & 280 & 27,10 & 17,10 & $15,4 \mathrm{a}$ & 20,20 & 4,70 & 4,30 & $5,5,0$ \\
\hline $\begin{array}{l}\text { Total-Salud-y-servicios- } \\
\text { socialeso }\end{array}$ & $26,8 \alpha$ & 27,30 & $26,7 \propto$ & 65,90 & $58,8 \alpha$ & $68,6 \mathrm{a}$ & $17,7 \mathrm{\alpha}$ & 16,10 & 18,30 \\
\hline $\begin{array}{l}\text { Enfermería } y \text {-atención } \cdot a \cdot \\
\text { enfermosa }\end{array}$ & $33,9 \alpha$ & 41,50 & 320 & 68,60 & 63,20 & $70,4 \propto$ & $23,3,0$ & 26,20 & 22,50 \\
\hline Otras-ciencias-de-la-Saludo & $27,1 \mathrm{a}$ & 28,30 & $26,5 \mathrm{a}$ & $60,7 \mathrm{xa}$ & $54,9 x$ & $63,7 \mathrm{x}$ & 16,$5 ;$ & 15,50 & 16,90 \\
\hline Trabajo-social-y orientacióno & 32,40 & $32,5 x$ & $32,4 a$ & $72,5 \approx$ & 64,90 & $74,1 \propto$ & $23,5 \times$ & 21,10 & $24,0,0$ \\
\hline Total-Serviciosa & $27,9 a$ & $26,8 \mathrm{a}$ & 29,40 & 43,90 & $43,2 \mathrm{a}$ & $44,8 \mathrm{a}$ & 12,20 & 11,60 & 13,20 \\
\hline Deportesa & $23,7 x$ & 23,90 & $* * * \alpha$ & 51,50 & 49,10 & $* * * a$ & 12,20 & $11,7 \alpha$ & $* * * a$ \\
\hline Turismo $y \cdot$ Hosteleríax & 31,60 & 33,10 & 30,80 & 38,90 & $33,8 x$ & $41,7 \mathrm{a}$ & $12,3,0$ & 11,20 & 12,90 \\
\hline
\end{tabular}

Fuente: Sistema Integrado de Información Universitaria. Ministerio de Educación. Nota: $\left.{ }^{* \star *}\right)$ Valores 0 o submuestras muy pequeñas. 
Diniz, Villar, Hernàndez \& Branco: Desajuste de trayectorias estudiantiles

Tabla 2 - Renovación, idoneidad y fluidez en la Universidad Federal de Paraíba en hombres y mujeres.

\begin{tabular}{|c|c|c|c|c|c|c|c|c|c|}
\hline \multirow{2}{*}{ TITULACION } & \multicolumn{3}{|c|}{ TASA-DE-RENOVACION(TR) } & \multicolumn{3}{|c|}{ TASA-DE-IDONEIDAD-(TI)a } & \multicolumn{3}{|c|}{ TASA.DE-FLUIDEZ-TFF $\alpha$} \\
\hline & Ambosa & Hombress & Mujeresa & Ambos $x$ & Hombreso & Mujeresa & Ambos $x$ & Hombrese & Mujeresa \\
\hline Agroindustriao & 16,30 & $16,5 x$ & 16,$0 ;$ & $20,5 x$ & $5,0,0$ & $36,8 \mathrm{a}$ & 3,30 & $0,8 \mathrm{o}$ & $5,9 \alpha$ \\
\hline Agronomíad & $14,7 \times$ & 15,30 & 13,20 & 10,30 & 14,30 & $* * * \alpha$ & $1,5 x$ & 2,20 & $* * * 0$ \\
\hline Ciencias-Agrariasa & 26,20 & $22,6 \alpha$ & 31,20 & 21,10 & 26,30 & $15,8 \mathrm{a}$ & $5,5 \propto$ & $6,0,0$ & $4,9 \alpha$ \\
\hline Biotecnología & 41,20 & $48,4 \alpha$ & 37,000 & 14,30 & $26,7 x$ & $5,0,0$ & 5,90 & $12,9 x$ & 1,90 \\
\hline $\begin{array}{l}\text { Ciencias-Biologicas--· } \\
\text { Campus-Io }\end{array}$ & $20,6 \mathrm{a}$ & $23,2 \mathrm{a}$ & $18,8 \mathrm{a}$ & $6,8 \mathrm{a}$ & 10,40 & 3,60 & $1,4 \alpha$ & $2,4 \alpha$ & $0,7 \times$ \\
\hline Ecologiad & $19,3 \mathrm{x}$ & $17,8 \mathrm{o}$ & 20,10 & $2,0 \times$ & $6,3 \mathrm{a}$ & $* * * \alpha$ & 0,40 & 1,10 & $* * * \alpha$ \\
\hline $\begin{array}{l}\text { Bachillerato-en-Educación- } \\
\text { Físicad }\end{array}$ & 14,20 & $14,6 \propto$ & $13,3 \alpha$ & $5,9 a$ & $* * * a$ & $20,0: x$ & 0,80 & $* * * \alpha$ & $2,7 \times$ \\
\hline $\begin{array}{l}\text { Formación-de maestros- } \\
\text { Educación-Física }\end{array}$ & 12,$9 ; 0$ & 11,$8 ;$ & $15,6 a$ & $15,8 \mathrm{a}$ & 4,20 & $35,7 \mathrm{a}$ & $2,0,0$ & $0,5 x$ & 5,60 \\
\hline Enfermería & 11,10 & $12,7 \times$ & $10,9: \alpha$ & 3,90 & $* * * \alpha$ & 4,60 & $0,4 \propto$ & $* * * a$ & $0,5 \alpha$ \\
\hline Farmaciaa & $12,2 \mathrm{o}$ & $14,5 \times$ & $10,9 \alpha$ & $4,0 \mathrm{\alpha}$ & $4,8 \mathrm{a}$ & $3,5 \mathrm{a}$ & $0,5 \mathrm{x}$ & $0,7 \mathrm{a}$ & $0,4 \alpha$ \\
\hline Logopediax & $15,7 \mathrm{a}$ & 18,90 & 15,20 & $29,7 \times$ & 14,30 & 33,30 & $4,7 \mathrm{x}$ & $2,7 \mathrm{o}$ & 5,10 \\
\hline Nutricióno & $13,7,0$ & 12,90 & 13,80 & 39,000 & 25,$0 ;$ & $40,5 x$ & 5,40 & 3,20 & 5,60 \\
\hline Terapia-Ocupacionala & 21,20 & 20,80 & 21,20 & 52,800 & $* * * 0$ & $61,3 a$ & 11,20 & $* * * a$ & $13,0,0$ \\
\hline Informáticaơ & 15,900 & $15,4 a$ & 19,40 & 11,10 & $10,5 \mathrm{a}$ & $14,3 \mathrm{o}$ & 1,80 & 1,60 & 2,80 \\
\hline $\begin{array}{l}\text { Ciencias-Biologicas--· } \\
\text { Campus-IIa }\end{array}$ & $22,5 x$ & $28,8 \mathrm{x}$ & $19,7 x$ & $12,5 \alpha$ & $10,5 x$ & $13,8 \mathrm{a}$ & 2,80 & $3,0,0$ & $2,7 \times 0$ \\
\hline Diseñoo & 24,10 & $33,8 x$ & 17,40 & 2,50 & 4,40 & $* * * 0$ & 0,60 & 1,50 & $* * * \alpha$ \\
\hline Ingeniería de Materiales $\alpha$ & $20,7 \times 0$ & 22,10 & 18,30 & 2,30 & 3,50 & $* * * \alpha$ & 0,50 & $0,8 a$ & $* * * a$ \\
\hline Estadisticaa & 33,80 & $28,6 \mathrm{a}$ & 42,90 & 11,10 & $12,5 x$ & 9,50 & 3,80 & 3,60 & 4,10 \\
\hline Matemáticaso & $34,0: 0$ & $34,8 a$ & 32,10 & 3,80 & 3,10 & $5,7 \times$ & 1,30 & 1,10 & $1,8 \mathrm{a}$ \\
\hline Matemática Computacionala & 47,10 & 46,20 & 50,$0 ;$ & 2,40 & $3,3,0$ & $* * * \alpha$ & 1,20 & $1,5 \mathrm{a}$ & $* * * a$ \\
\hline Química-Industrialo & 15,10 & $15,9 a$ & $14,1 \mathfrak{a}$ & $* * * a$ & $* * * a$ & $* * * \alpha$ & $* * * a$ & $* * * \alpha$ & $* * * a$ \\
\hline $\begin{array}{l}\text { Sistemas-de-Información-- } \\
\text { Campus-IVor }\end{array}$ & $24,4 \alpha$ & 22,40 & $35,7 \times$ & $* * * \alpha$ & $* * * \alpha$ & $* * * \alpha$ & $* * * \alpha$ & $* * * \alpha$ & $* * * \alpha$ \\
\hline Tecnología Alimentariaa & 29,40 & $21,6 \infty$ & 36,10 & $17,0: 0$ & $18,8 \mathrm{x}$ & $16,1 \mathrm{a}$ & $5,0,0$ & 4,10 & $5,8 \alpha$ \\
\hline $\begin{array}{l}\text { Tecnología-en-Producción- } \\
\text { de-Azúcar-y-Alcoholo }\end{array}$ & $27,9: 0$ & $26,8 a$ & 29,40 & $13,0: 0$ & $3,9, a$ & 25,$0 ; 0$ & 3,60 & $1,0,0$ & 7,40 \\
\hline Administracióno & $11,7, \alpha$ & 11,10 & 12,$5 ;$ & $8,7 a$ & 5,90 & 12,10 & $1,0,0$ & $0,7 \mathrm{a}$ & 1,$5 ;$ \\
\hline Artes-Visuales $\alpha$ & 27,20 & 31,70 & 24,20 & $10,7: a$ & 15,40 & $6,7: 0$ & 2,90 & 4,90 & 1,60 \\
\hline Biblioteconomía & 11,7 & 12,5 & 11,2 & 2,1 & $* * *$ & 3,3 & 0,2 & $* * *$ & 0,4 \\
\hline Ciencias Actuariales & 43,9 & 44,3 & 43,5 & 3,7 & 5,2 & 2,0 & 1,6 & 2,3 & 0,9 \\
\hline Ciencias Contables & 12,1 & 13,6 & 10,1 & 15,7 & 10,5 & 25,7 & 1,9 & 1,4 & 2,6 \\
\hline Ciencias de las Religiones & 17,4 & 23,1 & 10,9 & 2,3 & 3,2 & $* * *$ & 0,4 & 0,8 & $* * *$ \\
\hline Ciencias Económicas & 14,3 & 16,6 & 11,2 & 4,3 & 1,6 & 9,7 & 0,6 & 0,3 & 1,1 \\
\hline Filosofia & 27,8 & 27,9 & 27,5 & 6,2 & 6,5 & 5,3 & 1,7 & 1,8 & 1,5 \\
\hline Geografia & 12,2 & 12,6 & 11,6 & 15,9 & 23,3 & $* * *$ & 1,9 & 2,9 & $* * *$ \\
\hline Hotelería & 19,2 & 25,0 & 17,2 & 4,2 & 6,3 & 3,1 & 0,8 & 1,6 & 0,5 \\
\hline
\end{tabular}




\begin{tabular}{|l|c|c|c|c|c|c|c|c|c|}
\hline Periodismo & 36,1 & 38,1 & 34,9 & 23,1 & 6,3 & 34,8 & 8,3 & 2,4 & 12,1 \\
\hline Lenguas (Lengua Española) & 15,6 & 11,9 & 16,7 & 14,3 & 20,0 & 13,0 & 2,2 & 2,4 & 2,2 \\
\hline Lenguas - Campus IV & 60,0 & 66,7 & 58,3 & $* * *$ & $* * *$ & $* * *$ & $* * *$ & $* * *$ & $* * *$ \\
\hline $\begin{array}{l}\text { Lenguas Extranjeras } \\
\text { Aplicadas a las } \\
\begin{array}{l}\text { Negociaciones } \\
\text { Internacionales }\end{array}\end{array}$ & 28,9 & 31,0 & 27,4 & 10,0 & 15,4 & 5,9 & 2,9 & 4,8 & 1,6 \\
\hline $\begin{array}{l}\text { Pedagogía (Formación de } \\
\text { maestros de Enseñanza } \\
\text { Infantil y Primaria) }\end{array}$ & 14,2 & 21,0 & 13,3 & 12,3 & $* * *$ & 14,8 & 1,8 & $* * *$ & 2,0 \\
\hline $\begin{array}{l}\text { Pedagogía (Formacín de } \\
\text { maestros de Enseñanza } \\
\text { Infantil y Primaria)-Campus } \\
\text { III }\end{array}$ & 16,2 & 21,4 & 15,3 & 10,9 & $* * *$ & 13,5 & 1,8 & $* * *$ & 2,1 \\
\hline Psicopedagogia & 16,1 & 18,5 & 15,8 & 47,8 & $* * *$ & 53,7 & 7,7 & $* * *$ & 8,5 \\
\hline Radialismo & 39,3 & 42,6 & 35,1 & 24,2 & 25,0 & 23,1 & 9,5 & 10,6 & 8,1 \\
\hline $\begin{array}{l}\text { Tecnología en Gestión } \\
\text { Pública }\end{array}$ & 18,1 & 19,4 & 16,9 & 21,7 & 20,8 & 22,7 & 3,9 & 4,0 & 3,9 \\
\hline Traducción & 18,8 & 9,1 & 26,9 & $* * *$ & $* * *$ & $* * *$ & $* * *$ & $* * *$ & $* * *$ \\
\hline Turismo & 14,0 & 15,1 & 13,4 & 10,3 & $* * *$ & 17,4 & 1,4 & $* * *$ & 2,3 \\
\hline $\begin{array}{l}\text { Formación de maestros en } \\
\text { Música }\end{array}$ & 15,9 & 17,0 & 12,2 & 14,3 & 17,4 & $* * *$ & 2,3 & 3,0 & $* * *$ \\
\hline Bachillerato en Música & 19,5 & 17,4 & 25,8 & 12,5 & 18,8 & $* * *$ & 2,4 & 3,3 & $* * *$ \\
\hline Bachillerato en Teatro & 31,3 & 46,4 & 19,4 & 15,0 & 15,4 & 14,3 & 4,7 & 7,1 & 2,8 \\
\hline $\begin{array}{l}\text { Formación de maestros en } \\
\text { Teatro }\end{array}$ & 10,5 & 13,2 & 6,9 & $* * *$ & $* * *$ & $* * *$ & $* * *$ & $* * *$ & $* * *$ \\
\hline
\end{tabular}

Fuente: Servicio de Informática de la Universidade Federal de Paraíba. Nota: $\left.{ }^{(* \star}\right)$ Valores 0 o submuestras muy pequeñas.

La TF presenta un problema: al ser el producto de las otras dos tasas (TR y TI), podemos obtener un mismo resultado con factores inversos. Para evitar este problema es por lo que introducimos un Índice de Desajuste Académico (IDA), cuyo fundamento se explica a continuación.

Si representamos en unos ejes cartesianos TR (eje $\mathrm{x}$ ) y TF (eje y), tal como muestra el Diagrama 1, si se diera el máximo ajuste posible o el mínimo desajuste, los valores resultantes se dispondrían en la diagonal (d) que une los puntos $(0,0)$ y $(1,1)$. A mayor desajuste, el punto se alejaría de la diagonal, pero teniendo en cuenta la doble condición trivial de que a un valor $\mathrm{TR}=0$, corresponde un valor de $\mathrm{TF}=0$, y de que si $\mathrm{TF}=1$, necesariamente TR=1. Por ello, dado un par de valores de TR y TF, que denominaremos $\left(T R_{i}, T F_{j}\right)$, si trazamos una curva (I) que pase por ese punto y por los puntos $(0,0)$ y $(1,1)$, definiremos una superficie entre la curva y la diagonal, que denominaremos $G, y$ otra por debajo de la curva, que denominaremos S. Véase el Diagrama 1. 


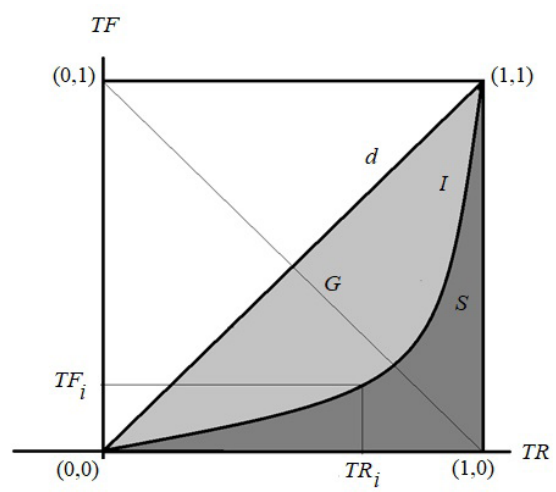

Diagrama 1 - Representación en ejes cartesianos de TR (eje x) y TF (eje y). Fuente: Elaboración propia.

Pues bien, determinaremos el Índice de Desajuste Académico (IDA) como la relación entre la superfície $G$ y la superfície $G+S$. Dado que:

$$
G+S=\frac{1}{2} \quad[4]
$$

Entonces:

$$
I D A=\frac{G}{G+S}=\frac{G}{\frac{1}{2}}=2 G=1-2 S \quad[5]
$$

Hay que hacer una explicación complementaria sobre la metodología. De las infinitas curvas que pueden pasar por los puntos $(0,0),\left(T R_{i}, T F_{j}\right)$ y $(1,1)$, se puede utilizar, por ejemplo, una curva parabólica y una curva exponencial. En el primer caso, la curva resultante tiene la condición de simetría; en el segundo, carece de tal condición. En ambos casos, el cálculo de la superficie G se realiza a partir de la superficie S, que se puede establecer con la integral definida de la función de la curva. En el caso de la curva parabólica, puede procederse a una traslación y un giro de los ejes cartesianos. Una hoja de cálculo habitual (como Excel) permite entonces trazar la curva y ofrecer su ecuación con la forma general: $y=a x^{2}+b x+c$. A continuación, se calcula la integral definida para calcular $S$ y luego IDA.

En el caso de la curva exponencial (procedimiento que desarrollaremos en otra ocasión), se puede utilizar una fórmula general, como, por ejemplo: $y=\left(2^{x}-1\right)^{z}$. Dado un par de valores, se calcula fácilmente z (con el recurso a logaritmos) y después se hace una simulación, proporcionando valores de x y obteniendo valores de y, en ambos casos entre 0 y 1 . Con estos valores se puede proceder a una representación, trazar 
una curva polinómica y calcular la integral definida para, igual que en el otro caso, establecer la superficie S y luego IDA.

En realidad, el procedimiento presentado aquí es una adaptación del conocido Índice o Coeficiente de Gini, que se suele utilizar en los estudios socioeconómicos para ofrecer una medida de la desigualdad. Aunque hay múltiples adaptaciones, el Índice de Gini en su versión tradicional ofrece el porcentaje de población acumulada (eje x) y el porcentaje de riqueza acumulada (eje y) de una determinada sociedad, lo que produce una curva semejante a la curva / del diagrama 1. El Índice se define como la proporción de la superficie $\mathrm{G}$ respecto de la suma $\mathrm{G}+\mathrm{S}$. Se puede apreciar pues la semejanza con nuestra formulación del IDA.

Considerando el carácter comparativo del estudio, hemos optado por el cálculo del índice de desajuste por la curva parabólica.

\section{Resultados}

Los resultados obtenidos en el caso de la Universidad de València (UV) son los recogidos para la cohorte de entrada de 2013-2014, y para la Universidad Federal de Paraíba (UFPB), para la cohorte 2013.1. La diversa nomenclatura tiene que ver con el hecho de que el año académico se organiza de manera diferente en los diversos hemisferios. En los datos se han utilizado las titulaciones de cuatro años, agrupadas según las fuentes oficiales. Los datos de las tablas 3 y 4 han sido ordenados (en orden decreciente de valores) por el valor de IDA (modelo de curva parabólica).

Tabla 3 - Índice de Desajuste Académico en la Universidad de València (por orden decreciente)

\begin{tabular}{|c|c|c|c|}
\hline \multirow{2}{*}{ TITULACIÓNa } & \multicolumn{3}{|c|}{ IDAa } \\
\hline & Ambosa & Hombresa & Mujeresa \\
\hline Ingenieríasa & $0,5629 a$ & 0,58300 & $0,5286 a$ \\
\hline Informáticax & $0,5472 \alpha$ & 0,55720 & $* * * a$ \\
\hline Ciencias-Físicas, químicas, geológicasa & $0,5237 \alpha$ & $0,4925 a$ & 0,5814 \\
\hline Humanidadesa & $0,4500 x$ & $0,4699: a$ & $0,4143 \mathrm{a}$ \\
\hline Matemáticas $\mathrm{y} \cdot$ Estadísticac & $0,4479 \propto$ & $0,4320 x$ & 0,47570 \\
\hline Otra Educación comercial-y·empresarialæa & $0,4416 a$ & 0,49170 & $0,4007 x$ \\
\hline Total-Ciencias-e-informáticao & 0,40410 & $0,4022 a$ & 0,40620 \\
\hline Artesa & $0,3961 \alpha$ & $0,4124 a$ & 0,38770 \\
\hline Derechood & $0,3879 \alpha$ & $0,4379 a$ & 0,35450 \\
\hline Turismo $y \cdot$ Hosteleríaa & $0,3758 a$ & $0,4237 \mathrm{a}$ & 0,3505 \\
\hline Economíad & $0,3704 \alpha$ & $0,4198 a$ & 0,30210 \\
\hline Total-Artes $y$-humanidades & $0,3616 \alpha$ & $0,4033 \mathrm{a}$ & $0,3358 \%$ \\
\hline Otras-Ciencias-sociales $\cdot \mathrm{y} \cdot \mathrm{del}$-comportamiento & $0,3500 \%$ & 0,37410 & 0,33360 \\
\hline Lenguasa & $0,3366 \alpha$ & $0,3675 \ldots$ & $0,3267 \mathrm{a}$ \\
\hline Periodismo e-informacióna & $0,3339 \alpha$ & 0,36720 & 0,31410 \\
\hline Total Serviciosa & $0,3251 \alpha$ & $0,3273 a$ & 0,3226 \\
\hline Total-Ciencias - sociales $y$-derechoo & $0,3225 \alpha$ & 0,36930 & 0,2936 \\
\hline
\end{tabular}




\begin{tabular}{|c|c|c|c|}
\hline Administración·y·gestión-de-empresasó & $0,3115 \alpha$ & $0,3652 \alpha$ & 0,26190 \\
\hline Totala & $0,2986 a$ & $0,3664 \alpha$ & 0,25740 \\
\hline Ciencias $\cdot d e \cdot l a \cdot v i d a d a$ & $0,2951 a$ & $0,2878 a$ & $0,3007 x$ \\
\hline Psicología & $0,2787 \propto$ & $0,3121 \alpha$ & 0,26650 \\
\hline Deportesa & $0,2599 a$ & $0,2768 \alpha$ & $* * * \alpha$ \\
\hline Otras ciencias de-la-Saluda & $0,2084 a$ & $0,2485 a$ & $0,1886 a$ \\
\hline Otra-Formación de personal-docente $y \cdot$ ciencias de-la-educación & $0,1988 \alpha$ & $0,3232 \alpha$ & $0,1795 \alpha$ \\
\hline Total-Salud-y-servicios-socialesor & $0,1761 \propto$ & $0,2206 a$ & $0,1603 \alpha$ \\
\hline Técnicas audiovisuales $y$-medios de comunicacióno & $0,1743 \alpha$ & 0,18420 & 0,16740 \\
\hline Enfermería·y-atención-a·enfermosa & $0,1739 \alpha$ & $0,2273 \alpha$ & $0,1589 \alpha$ \\
\hline Formación-de-docentes-de-enseñanza-infantilo & $0,1623 \alpha$ & $* * * \alpha$ & $0,1603 \alpha$ \\
\hline$\underline{\underline{T}} \underline{\underline{T}} \cdot$ Educaciónd & $0,1614 \alpha$ & $0,2115 a$ & 0,14940 \\
\hline Trabajo-social-y orientacióna & $0,1475 \alpha$ & $0,1940 x$ & 0,13780 \\
\hline Formación de docentes de enseñanza primaria & $0,1420 \alpha$ & $0,1903 a$ & 0,12110 \\
\hline
\end{tabular}

Fuente: Sistema Integrado de Información Universitaria. Ministerio de Educación.

Nota: Se han sombreado los valores superiores en la comparación hombres-mujeres. $\left.{ }^{* \star *}\right)$ Valores no pertinentes por valores 0 o submuestras muy pequeñas en los cálculos de TR, TI y TF.

Según los datos recogidos en la tabla 3 para el caso de la UV, las titulaciones con mayores desajustes son, por un lado, las Ingenierías, la Informática, las Ciencias Físicas, químicas y geológicas, y las Matemáticas y Estadística, y, por otra parte, las Humanidades y las Artes. Las ciencias con menor índice de desajuste son ciencias sociales y de la salud, concretamente formación de docentes (que en la legislación española se considera una ciencia social), trabajo social y enfermería; también técnicas audiovisuales y medios de comunicación, deportes y psicología.

Tabla 4 - Índice de Desajuste Académico en la Universidad Federal de Paraíba (por orden decreciente)

\begin{tabular}{|c|c|c|c|}
\hline \multirow{2}{*}{ TITULACIÓNo } & \multicolumn{3}{|c|}{ IDA } \\
\hline & Ambosa & Hombreso & Mujeresa \\
\hline Matemática Computacionaló & $0,8368 \mathrm{a}$ & $0,8189 x$ & $* * * a$ \\
\hline Ciencias-Actuariales $-a$ & 0,8016 & $0,7835 a$ & 0,82310 \\
\hline Matemáticaso & $0,7505 a$ & $0,7635 a$ & $0,7162 \mathrm{a}$ \\
\hline Diseñox & 0,72350 & $0,7420 \mathrm{x}$ & $* * * \alpha$ \\
\hline Ingeniería-de Materialesa & $0,7122 \%$ & $0,7026 a$ & $* * * a$ \\
\hline Ecologiaa & $0,7105 \propto$ & 0,6496 & $* * * a$ \\
\hline Ciencias de las-Religiones $\alpha$ & $0,6992 \alpha$ & $0,7098 a$ & $* * * a$ \\
\hline Filosofiad & 0,69130 & 0,6872 a & 0,7016 \\
\hline Hoteleríac & $0,6815 \alpha$ & $0,6782 \alpha$ & $0,6871 \mathrm{a}$ \\
\hline Biblioteconomía & $0,6800: 0$ & 0,7111 a & 0,66200 \\
\hline Ciencias Económicaso & $0,6617 \mathrm{a}$ & $0,7052 \alpha$ & $0,5849 a$ \\
\hline Farmacia & $0,6570: 0$ & $0,6558 \mathrm{a}$ & $0,6594 a$ \\
\hline Estadísticad & $0,6568 a$ & $0,6177 a$ & $0,7195 a$ \\
\hline Enfermeriad & 0,65410 & $* * * a$ & 0,64540 \\
\hline Biotecnologíar & $0,6537 \mathrm{a}$ & $0,5564 \alpha$ & $0,7488 a$ \\
\hline
\end{tabular}




\begin{tabular}{|c|c|c|c|}
\hline $\begin{array}{l}\text { Lenguas-Extranjeras -Aplicadas-a-las-Negociaciones - } \\
\text { Internacionalesa }\end{array}$ & $0,6483 \alpha$ & $0,5951 \alpha$ & 0,69310 \\
\hline Bachillerato-en-Educación-Físicad & $0,6405 a$ & $* * * a$ & $0,4831 \alpha$ \\
\hline Artes-Visualeso & $0,6328 \alpha$ & $0,5983 \alpha$ & $0,6698 \alpha$ \\
\hline Tecnología-en-Producción-de-Azúcar·y·Alcohola & $0,6086 \alpha$ & 0,71710 & $0,4901 x$ \\
\hline Bachillerato-en-Teatro: & 0,60080 & $0,6677 \alpha$ & $0,5625 \alpha$ \\
\hline Administracióno & 0,59780 & 0,62960 & 0,56250 \\
\hline Ciencias-Biologicas--Campus-IIo & $0,5938 \alpha$ & $0,6419 a$ & $0,5689 \alpha$ \\
\hline Agronomíar & 0,58950 & $0,5480 \alpha$ & $* * * \alpha$ \\
\hline $\begin{array}{l}\text { Pedagogía-(Formación-de maestros de-Enseñanza-Infantil-y·Primaria)- } \\
\text {-Campus-IIlo }\end{array}$ & 0,58890 & $* * * \alpha$ & $0,5563 \alpha$ \\
\hline Turismoa & 0,58810 & $* * * 0$ & 0,50910 \\
\hline Informáticå & $0,5849 a$ & 0,58980 & $0,5625 x$ \\
\hline Bachillerato-en-Músicaơ & $0,5825: 0$ & $0,5088 x$ & $* * * a$ \\
\hline Tecnología-Alimentariaa & $0,5707 \alpha$ & 0,52320 & 0,60880 \\
\hline Pedagogía-(Formación-de maestros de-Enseñanza-Infantil-y-Primaria): & $0,5651 \propto$ & $* * * \alpha$ & 0,53520 \\
\hline Formación-de maestros-en-Músicad & 0,55010 & 0,52120 & $* * * a$ \\
\hline Lenguas-(Lengua·Española) $\alpha$ & $0,5487 \alpha$ & $0,4785 \alpha$ & 0,56600 \\
\hline Radialismoo & 0,53780 & 0,545000 & 0,53150 \\
\hline Periodismox & $0,5357 \propto 0$ & $0,7375 x$ & 0,42160 \\
\hline Formación-de maestros -Educación-Física-a & 0,52400 & $0,6534 \alpha$ & 0,35300 \\
\hline Ciencias Contables $\alpha$ & 0,52250 & 0,58440 & $0,4207 x$ \\
\hline Geografiax & $0,5204 \alpha$ & $0,4492 a$ & $* * * a$ \\
\hline Ciencias-Agrariasa & $0,5166 \mathrm{a}$ & $0,4537 \alpha$ & $0,5915 x$ \\
\hline Agroindustriaa & $0,4875 a$ & $0,6606 a$ & $0,3455 \mathrm{a}$ \\
\hline Tecnología-en-Gestión-Públicad & 0,48160 & $0,4946 a$ & $0,4684 a$ \\
\hline Logopediax & 0,40220 & $0,5606 a$ & $0,3707 x$ \\
\hline Nutricióno & 0,32320 & 0,43500 & $0,3122 . a$ \\
\hline Psicopedagogiad & $0,2670 x$ & $* * * 0$ & 0,22890 \\
\hline Terapia-Ocupacionala & $0,2460 \alpha$ & $* * * \alpha$ & $0,1931 \alpha$ \\
\hline Química-Industrialo & $* * * \alpha$ & $* * * 0$ & $* * * a$ \\
\hline Sistemas-de-Información--Campus-IVo & $* * * 0$ & $* * * \alpha$ & $* * * a$ \\
\hline Lenguas-Campus-IVo & $* * * 0$ & $* * * \alpha$ & $* * * a$ \\
\hline Traduccióno & $* * * a$ & $* * * a$ & $* * * a$ \\
\hline Formación-de maestros en-Teatrox & $* * * a$ & $* * * \alpha$ & $* * * \alpha$ \\
\hline
\end{tabular}

Fuente: Servicio de Informática de la Universidade Federal de Paraíba.

Nota: Se han sombreado los valores superiores en la comparación hombres-mujeres.. $\left(^{* \star \star}\right)$ Valores no pertinentes por valores 0 o submuestras muy pequeñas en los cálculos de TR, TI y TF.

Como puede observarse en la tabla 4, en el caso de la UFPB se repite aproximadamente el patrón descrito en el caso de la UV. Presentan IDA superiores las carreras de ciencias básicas e ingenierías (como Matemática Computacional, Matemáticas, Ingeniería de Materiales, etc.), así como humanidades como las Ciencias de las Religiones y Filosofía. También en el caso brasileño los menores IDA corresponden a carreras relacionadas con la educación, el cuidado sanitario o los medios de comunicación. Hay que destacar también que las Ciencias Agrarias y el grado relacionado con la Agroindustria (ausentes en la UV) presentan valores del IDA relativamente bajos. 
Respecto a la comparación entre ambas universidades, aunque las agrupaciones de titulaciones o grados son diferentes, el IDA permite una primera aproximación. Así, en este sentido, se pueden elabora comparaciones, tal y como recogemos a modo de ejemplo en la siguiente tabla.

Tabla 5 - Com5paraciones de IDA entre la UV y la UFPB

\begin{tabular}{|c|c|c|}
\hline TITULACIÓNa & IDA-UVa & IDA-UFPB $a$ \\
\hline Ingeniería $a$ & $0,5629 a$ & 0,7122 a \\
\hline Informáticad & 0,5472 a & $0,5849 a$ \\
\hline Matemáticas $\mathrm{a}$ & $0,4479 a$ & 0,7505 व \\
\hline Economía & $0,3704 \alpha$ & $0,6617 \not$ \\
\hline Turismod & 0,3758 व & 0,5881 a \\
\hline Enfermeríad & 0,1739 ㅁ & 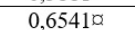 \\
\hline
\end{tabular}

Fuente: Elaboración propia.

Como puede apreciarse, aunque el orden del IDA presenta patrones semejantes en ambos centros universitarios, por lo que se refiere a los valores concretos en cada caso, Ia UFPB presenta valores de IDA superiores a la UV. Se podría ampliar esta conclusión a otros ejemplos recogidos en las tablas 3 y 4.

\section{Discusión y conclusiones}

Este trabajo muestra que los habituales indicadores de renovación e idoneidad permiten la construcción de un indicador más preciso, el Índice de Desajuste Académico, de una manera comprehensiva e integrada, que permite una aproximación cuantitativa al ajuste o desajuste de las trayectorias académicas.

Si nos detenemos a observar este índice según el orden de mayor a menor desajuste (según el modelo parabólico) en las áreas de estudio, los resultados aportan, en ambos casos, evidencias sobre el comportamiento heterogéneo dentro de la universidad, ya que se aprecian diferencias considerables entre las distintas agrupaciones de áreas de estudio lo que plantea un reto para las instituciones universitarias que ya hemos señalado en trabajos anteriores (Villar, A.; Hernàndez, F. J. \& García-Ros, R., 2017) con otras evidencias sobre rendimiento y trayectorias académicas. Este reto se concretaría en que las universidades deberían, primeramente, ser conscientes y conocer en profundidad sus divergencias internas y la heterogeneidad entre áreas de estudio para poder proponer intervenciones diferenciadas.

Las trayectorias académicas presentan ajustes académicos distintos en hombres y mujeres. A partir de nuestros cálculos puede observarse que los hombres suelen presentar valores de IDA superiores a las mujeres, tanto en el caso de la UV como en el caso de la UFPB. La tendencia es mucho más clara en aquellos grados con IDA bajo.

Consideramos que los indicadores educativos deben de ir revisándose para atender las transformaciones del sistema educativo. Como ya se ha apuntado, el fenómeno 
de la expansión universitaria ha comportado una mayor heterogeneidad en el perfil del estudiante y, con ello, se presentan situaciones y condiciones más diversas, como por ejemplo el aumento de estudiantes que combinan estudios y trabajo o un incremento de estudiantes de mayor edad o con responsabilidades familiares. Esta reflexión final se corresponde con la aspiración de avanzar hacia una universidad más inclusiva que atienda la diversidad de situaciones y condiciones en su estudiantado. Los indicadores educativos son una herramienta potencial para avanzar en la inclusión.

\section{Agradecimientos:}

Este manuscrito forma parte de un proyecto de investigación "Mapa da Educação Superior na Paraíba: análise do setor público e privado", cuya referencia es: CHAMADA UNIVERSAL MCTI/ CNPq No 01/2016.

\section{Referencias}

Ariño, A. V. (dir.), Hernández, M., Llopis, R., Navarro, P. Terrenina, B. (2008). El oficio de estudiar en la universidad: compromisos flexibles. València: Universitat de València, Servei de Publicacions.

Brooks, R. (2018). The construction of higher education students in English policy documents, British Journal of Sociology of Education, 39 (6), 745-761. DOI: 10.1080/01425692.2017.1406339

Brooks, R. (2019). The construction of higher education students within national policy: a crossEuropean comparison. A Journal of Comparative and International Education. 0 (0), 1-20. DOI: 10.1080/03057925.2019.1604118

Castelo Branco, U. V., Nakamura, P. H. \& Jezine, E. (2017). Permanência na educação superior no Brasil: Construção de uma escala de medida. Revista de la Asociación de Sociología de la Educación (RASE), 10 (2), 209-224.

Chaves, V. L. J; Maués, O. C.; Hage, S. M. (orgs.) (2016). Expansão privado-mercantil da educação superior no Brasil. Campinas: Mercado das Letras.

Daza, L., Troiano, H. y Elias, M. (2019). La transición a la universidad desde el bachillerato y desde el CFGS. La importancia de los factores socioeconómicos. Papers, 104 (3), 425-445. DOI: 10.5565/ rev/papers.2546

Dias Sobrinho, J. \& Brito, M. R. F. (2008). La educación Superior en Brasil: principales tendencias y desafios. Avaliação, 13 (2), 487-507.

Erlich, V. (2004). The "New" Students. The Studies and Social Life of French University Students in a Context of Mass Higher Education. European Journal of Education, 4 (39), 485-495.

Jezine, E. \& Bittar, M. (orgs.). (2013). Políticas de Educação Superior no Brasil: expansão, acesso e igualdade social. João Pessoa: UFPB. 
Nakamura, P. H., Castelo Branco, U. V. \& Jezine, E. (2016). Escala para Avaliação da Permanência Discente.

Segrera, F. L. (2008). Tendencias de la Educación Superior em el mundo y en América Latina y el Caribe. Avaliação, 13 (2), 267-291.

Sguissardi, V. (2015). Educação Superior no Brasil. Democratização ou massificação mercantil? Educação Social, 36 (133), 867-889.

Villar, A.; Hernàndez, F. J. \& García-Ros, R. (2017). Relocating within the university. Proposal of a relocation rate based on a study of educational trajectories. Relieve, 23(1), art. 5. DOI: 10.7203/ relieve.23.1.9059.

Villar, A., Vieira, M., Hernàndez, F. J. \& Nunes, A. (2012). Más que abandono de estudios, trayectorias de reubicación universitaria. Aproximación comparada al caso español y portugués. Revista Lusófona de Educação, 21, 139-162. [Disponível em https://revistas.ulusofona.pt/index.php/rleducacao/ article/view/3085. Consultado em 20-07-2019].

Adriana Valéria Santos Diniz

(Universidade Federal de Paraíba, Brasil) Doutora pela Universidade de Valência - Espanha. Professora Adjunta IV da Universidade Federal da Paraíba. Coordenadora do Programa de Pós-Graduação em Gestão das Organizações Aprendentes (MPGOA). Coordenadora do Projeto Universal - Mapa da Educação Superior na Paraíba - CNPQ.

E-mail: adrianavsdiniz@hotmail.com ORCID: https://orcid.org/0000-0002-2720-2433

Alícia Villar-Aguilés

(Universitat de València, Spain) Doutora em Sociologia (Universidade de Valência). Professora da Universidade de Valência e secretària do Instituto Universitário de Criatividade e Innovação Educativa. Directora de la Revista de Sociología de la Educación-RASE.

E-mail: alicia.villar@uv.es ORCID: http://orcid.org/0000-0003-1748-0234

Francesc J. Hernàndez (Universitat de València, Spain) Doutor em Sociologia (Universidade de Valência). Professor da Universidade de Valência. Director del Instituto Universitário de Criatividade e Innovação Educativa. E-mail: Francesc.J.Hernandez@uv.es ORCID: https://orcid.org/0000-0001-5229-2998 


\section{Uyguaciara Veloso Castelo Branco} (Universidade Federal de Paraíba, Brasil)

Doutora em História (Universidade Federal de Pernambuco - UFPE). Pós-Doutora em Sociologia (Universidade de Valência - UV). Professora Associada IV da Universidade Federal da Paraíba. Integra a Pesquisa Mapa da Educação Superior na Paraíba - CNPq e o Grupo de Estudos em Educação Superior e Sociedade (GEES). E-mail: uyguaciara@gmail.com ORCID: https://orcid.org/0000-0003-3595-1415

Correspondência:

Adriana Valéria Santos Diniz Universidade Federal da Paraíba - Campus I Centro de Educação (CE) Programa de Pós-Graduação em Gestão das Organizações Aprendentes (MPGOA) Cidade Universitária - CEP 58051-900 - João Pessoa-PB-Brasil

Data de submissão: Setembro 2019 Data de avaliação: Dezembro 2019

Data de publicação: Novembro 2020 